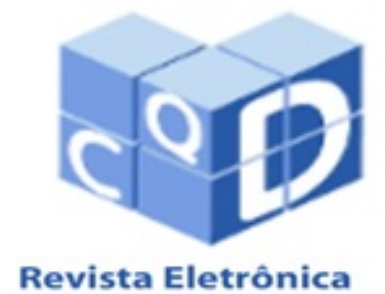

Paulista de Matemática

ISSN 2316-9664

Volume 10, dez. 2017

Edição Ermac

Marlon Paulo de Melo Wolff

Faculdade Estácio de São Luís marlonmelowolff@gmail.com

Aleff Viegas Abreu

Faculdade Estácio de São Luís aleff.v.abreu@gmail.com

\section{Tomada de decisão no setor de celulose e papel utilizando progamação por metas ponderadas}

Decision making in the pulp and paper sector using weighted goal programming

\begin{abstract}
Resumo
Neste trabalho é desenvolvido um estudo sobre uma metodologia da Pesquisa Operacional (PO) denominada Programação por Metas (PM) cuja principal característica reside na modelagem e análise de problemas de otimização considerando múltiplas metas e objetivos, mesmo havendo sentido conflitante entre estas, tais como, reduzir custo e elevar produtividade. Uma aplicação da PM será construída contemplando o setor produtivo associado a celulose e papel, abordando especificamente a produção de papéis sanitários, onde a matéria prima advém tanto de papel reciclado quanto de fornecedores de celulose. O resultado da PM no estudo de caso considerado permite ao tomador de decisão uma melhor avaliação referente à performance das variáveis econômicas, de produção e de sustentabilidade envolvidas no processo produtivo. Palavras-chave: Tomada de decisão, Progamação por metas, Indústria de papéis.
\end{abstract}

\begin{abstract}
In this work is developed a study about a methodology of the Operational Research called goal programming (GP), whose the main characteristic resides in modelling and analysis of optimization considering many goals and objectives, even though there are a conflicting sense between these, such as, reducing costs and raising productivity. A GP application Will be developed contemplating the productive sector associated with pulp and paper, specifically addressing the production of sanitary paper, where the raw material comes from both recycled paper and pulp suppliers. The result of GP in the case study considered enables the decision maker a better evaluation regarding performance of economic, production and sustainability variables, involved in the productive process.
\end{abstract}

Keywords: Decision making, Goal programming, Paper industry. 


\section{Introdução}

Uma característica inerente as indústrias e prestadoras de serviço em geral, é a necessidade de atingir diferentes metas simultaneamente, a Programação por Metas ou Goal Programming é uma técnica da Pesquisa Operacional com perspectiva multiobjetivo em relação aos problemas de tomada de decisão nestes setores. Como considera Ragsdale (2011, p. 306), em relação a Programação por Metas (PM), "é bem possível que uma variedade de funções objetivos possa ser proposta para o mesmo problema de otimização", sendo assim, trata-se de uma técnica da Pesquisa Operacional que apresentará um conjunto de soluções que servirá de apoio a tomada de decisão.

A PM é considerada ponderada quando a expressão matemática que descreve a função objetivo incorpora, além do conjunto de variáveis de desvios típicas dessa metodologia, valores de ponderação associadas a preferências específicas por parte do tomador de decisão em relação ao cumprimento das metas do problema formulado. Através destes parâmetros é possível reordenar a função objetivo para níveis de otimização mais desejáveis.

Nesse contexto, percebe-se que a PM esta ligada a outras técnicas de otimização da Pesquisa Operacional, contudo, a PM apresentará um grupo metas e objetivos a serem alcançados, segundo Sen e Nandi (2012, p. 2), "a progamação por metas é uma das mais antigas técnicas de decisão multicritérios com o objetivo de aperfeiçoar varias metas, e ao mesmo tempo minimizar os desvios da cada um dos objetivos do alvo desejado".

Neste trabalho é desenvolvida uma aplicação da PM ponderada no setor produtivo da celulose e papel, fornecendo um tratamento otimizado a tomada de decisão em relação as atividades de produtividade, de custo e de sustentabilidade que permeiam desenvolvimento fabril de indústrias deste setor. As técnicas de otimização aplicadas a indústria são bem exploradas na literatura.

Nesse cenário, alguns trabalhos como : estudos sobre o problema de corte de estoque unidimensional com aplicação na indústria de papel e otimização do problema de dimensionamento de lotes integrado ao problema de corte de estoque bidimensional em uma indústria de papel, apresentam possibilidades de otimização relacionadas ao planejamento e programação da produção em indústrias de papel e também a melhoria nos dimensionamentos dos lotes e problemas de cortes de estoque.

Assim sendo, problemas associados a otimização da produção de papel são encontrados em diversas aplicações, por exemplo, Bittencourt (2004) considera a melhoria no processo de fabricação de celulose e papel, por meio da caracterização dendométrica das árvores e da determinação da massa específica em diferentes idades da matéria prima. Nas proximas seções serão apresentadas respectivamente, dados do setor da celulose e papel, as características matemáticas da Progamação por Metas e posteriormente a simulação computacional com a análise dos resultados alcançados.

\section{Setor de celulose e papel}

A celulose é um produto da complexa organização industrial do setor florestal, sendo considerado no Brasil produto madeireiro, juntamente com o carvão vegetal, madeira serrada e outros. A produção de celulose deriva principalmente das florestas de eucalipto e pinus. Segundo Nali, Ribeiro e Da Hora (2016, p. 257), "o Brasil tem a maior produtividade florestal do mundo, tanto no cultivo de pinus quanto no de eucalipto". Com volume médio anual de $44 \mathrm{~m} 3 / \mathrm{ha} / \mathrm{ano}$ em 
produção de eucalipto e cerca de 1,59 milhão de hectares de pinus, o Brasil dispara na frente de outros grandes centros de produção florestal, como África do Sul, Chile, Portugal e Espanha.

Dados da Indústria Brasileira de Árvores (2015) revelam que do total de 7,74 milhões de hectares de árvores plantadas em um cenário internacional, o Brasil se mantém como quarto produtor mundial de celulose, tendo a produção brasileira registrado em 2015 um crescimento de 4,6\% de janeiro a outubro, passando de 13,6 milhões de tonelada em 2014 para 14,2 milhões de toneladas em 2015. Apenas cinco empresas concentram cerca de $80 \%$ da capacidade produtiva. Em 2009, somente a Fibria S.A. detinha 36,6\% do percentual de produção. Segundo dados recentes a indústria de papel tem crescido em volume de vendas principalmente em exportações, tendo em vista que entre 2014 e 2015 houve um aumento de cerca de 8,6\% no volume de exportação, a China continua sendo um dos principais clientes do comércio de celulose nacional.

Dados demonstram que a Europa ainda é o maior cliente da indústria brasileira, posteriormente a China foi responsável por cerca de 33,2\% da receita total de vendas de papel e celulose, seguida pela América do Norte (SILVA; BUENO; NEVES, 2015). Em uma cadeia nacional apesar das incertezas comercias e de um cenário recessivo, esse mercado tem se mantido equilibrado, mesmo que a receita tenha sido em 2015 4,5\% menor do que em 2014, os níveis de exportação tem se mostrado um bom agente de equilíbrio no mercado.

Problemas logísticos e no gerenciamento das cadeias produtivas de papel e celulose, se apresentam como grandes desafios as indústrias brasileiras, principalmente a nível internacional, sendo assim, seja por modais de transporte rodoviário, ferroviário ou marítimo, é necessário um planejamento estratégico com o objetivo de minimizar problemas relacionados a cadeia produtiva e ao escoamento desses produtos.

\section{Progamação por Metas Ponderadas}

O modelo de Progamação por Metas é formulado de forma bastante similar a um modelo de Programação Linear, contendo, ainda assim, algumas diferenças em relação às funções objetivo que são inseridas com prioridades desejadas. Outra abordagem para a Programação por Metas é declarar os objetivos como restrições, além das restrições normais do problema. A função objetivo é, portanto, montada para minimizar variáveis de desvio em relação às metas desejadas pelo gestor. Aos desvios apresentados na função objetivo são fornecidas ponderações dadas de acordo com a visão do gestor sobre o problema.

Portanto, em relação a outras técnicas da pesquisa operacional tais como, Programação Linear, Programação Linear Inteira, a Programação por Metas apresenta adicionalmente variáveis de desvio $\left(d_{i}^{-}+d_{i}^{+}\right)$, que são marcadas por expressar as quantidades de falta ou excesso da solução em relação as metas, que por sua vez traduzem os objetivos específicos do problema. Além dos desvios, as ponderações $\left(w_{i}^{-}+w_{i}^{+}\right)$representam parâmetros que são utilizados para moderar as variáveis de desvios do problema, ou seja, podemos privilegiar algumas metas em relação a outras dependo da situação apresentada tornando alguns valores dentro da solução indesejáveis ou não.

A seleção dos valores de ponderação para a função objetivo da PM não é o principal foco deste trabalho, mas acentua-se que possibilidades (pesquisas futuras) podem ser devidamente tratadas, a saber :

- Através de uma conveniente interação estratégica tipificada pela Teoria dos Jogos, onde 
teríamos as metas, ou bloco de metas, como agentes de decisão e estratégias sendo dadas pelos valores de $\left(w_{i}^{-}+w_{i}^{+}\right)$

- Execução de uma rotina computacional permitiria uma seleção conveniente para os valores das ponderações.

- O processo mais geral ocorre por tentativas e refinamento da solução mínima.

As Equações (1)-(3) representam a formulação matematica da Progamação por Metas Ponderadas, conforme estão descritas abaixo :

$$
\begin{gathered}
\text { MIN }: \sum_{i=1}^{m} \frac{1}{h_{i}}\left(w_{i}^{+} d_{i}^{+}+w_{i}^{-} d_{i}^{-}\right) . \\
f_{i}(x)-d_{i}^{-}+d_{i}^{+}=g_{i}, i=1,2 \ldots n . \\
d_{i}^{+}, d_{i}^{-} \geq 0, i=1,2, \ldots, n .
\end{gathered}
$$

Sendo $\frac{1}{h_{i}}$ o fator que permite normalizar os desvios percentuais e tendo em vista que as metas são, em geral, qualificadas de forma diferenciada, por exemplo, uma meta pode referir-se a quantidade de dinheiro enquanto outra a pessoas. O termo $h_{i}$ representa o valor ótimo da meta i, onde o fluxo de desenvolvimento da metodologia considera uma lógica de tratamento das metas por meio das variáveis de desvio e ponderações.

A função objetivo descrita na Equação 1, está sujeita as retrições apresentadas nas Equações (2)-(3), na Equação 2, $f_{i}(x)$ é a representação de varias funções objetivos, onde para cada $f_{i}(x)$ temos uma meta $g_{i}$, as metas são delimitadas para cada objetivo i. As primeiras variáveis servem de sensores em relação ao cumprimento das metas, enquanto as segundas operam como instrumentos de controle do modelo de solução, permitindo a varredura de soluções mais desejáveis.

\section{Estudo de Caso}

Esta seção é dedicada ao estudo da Programação por Metas através de uma aplicação em uma empresa de produção de papéis sanitários, onde os conjuntos de requisitos são descritos na Seção 4.1 para aplicação na Seção 4.2.

\subsection{Descrição do processo produtivo da empresa}

A situação problema em que será desenvolvida a Programação por Metas Ponderadas contempla a cadeia produtiva da empresa, a seguir destaca-se a descrição e seu processo produtivo. 
1. Seu processo produtivo é descrito no fluxograma da Figura 1 abaixo.

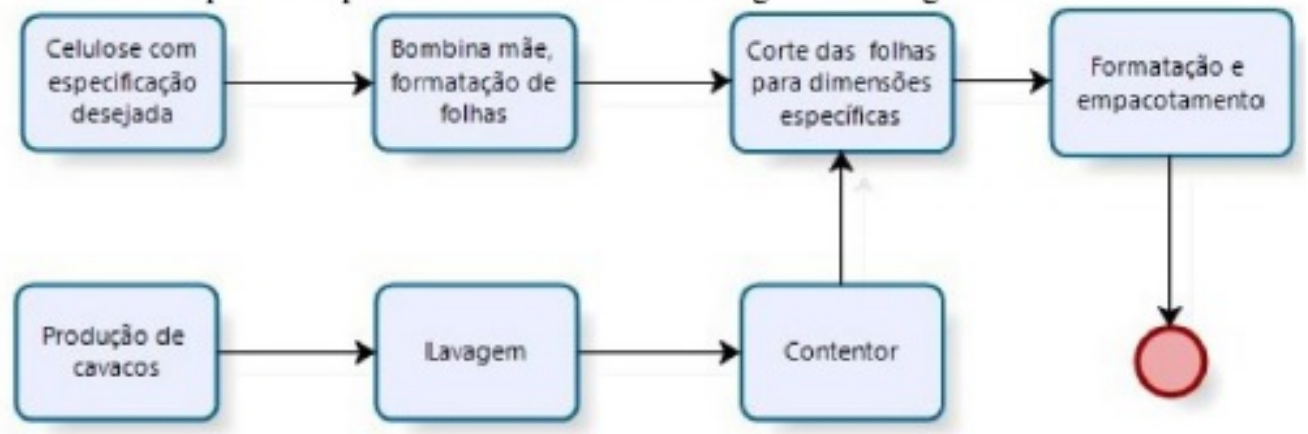

Figura 1 - Fluxograma do processo produtivo da empresa

Fonte - Elaborado pelos autores

A empresa atua com produção de papel e celulose, sendo considerada uma indústria de bens de consumo com certa referência no distrito industrial onde estar localizada. A gerência executiva da empresa avalia os resultados de produtividade e venda, verifica que o mercado tem apresentado certo desaquecimento e pretende viabilizar um planejamento satisfatório que atenda três aspectos de desempenho, a saber : produtivo, financeiro e sustentável. A partir de análises de mercado e dos processos internos, a gerência estabeleceu como metas para o próximo mês as quantidades de produtos descritas abaixo :

- META 1: Produzir aproximadamente 8000 unidades de papel toalha PIT $\left(X_{1}\right)$

- META 2: Produzir aproximadamente 2265 unidades de papel toalha PTB $\left(X_{2}\right)$

- META 3: Produzir aproximadamente 1119 unidades de papel toalha PIM $\left(X_{3}\right)$

- META 4: Produzir aproximadamente 860 unidades de papel big roll PRB $\left(X_{4}\right)$

- META 5: Produzir aproximadamente 423 unidades de papel lençol H PLH $\left(X_{5}\right)$

- META 6: Produzir aproximadamente 19000 unidades de papel higiênico PH $\left(X_{6}\right)$

- META 7: Produzir aproximadamente 2110 unidades de papel toalha PT $\left(X_{7}\right)$

- META 8: Alcançar uma receita liquida de aproximadamente 380.000 reais $\left(X_{8}\right)$

- META 9: Custo total de produção de aproximadamente 200.000 reais $\left(X_{9}\right)$

- META 10: Consumir aproximadamente 95.000 litros de agua $\left(X_{10}\right)$

\subsection{Aplicação da Progamação por Metas em uma empresa de produção de papéis sanitários}

O processo de modelagem matemática dentro da Programação por Metas é relativamente similar ao utilizado pela Programação Linear, por isso, podemos considerar as metas como restrições do problema. As metas podem ser escritas matematicamente como restrições flexíveis, 
conforme as Equações (4)-(13). A seguir são explanadas cada tipo de papel e sua respectiva meta, juntamente com o valor de cada receita, custo e consumo de água por unidade de cada tipo de papel.

Os números que antecedem cada variável de decisão nas Equações (11)-(13), representam as receitas unitárias, custo de produção unitário e consumo unitário respectivamente, para cada tipo de papel.

$$
\begin{gathered}
X_{1}+d_{1}^{-}+d_{2}^{+}=8000 \\
X_{2}+d_{2}^{-}+d_{2}^{+}=2265 \\
X_{3}+d_{3}^{-}+d_{3}^{+}=1119 \\
X_{4}+d_{4}^{-}+d_{4}^{+}=860 \\
X_{5}+d_{5}^{-}+d_{5}^{+}=423 \\
X_{6}+d_{6}^{-}+d_{6}^{+}=19000 \\
X_{7}+d_{7}^{-}+d_{7}^{+}=2110 \\
12 X_{1}+12 X_{2}+15 X_{3}+10 X_{4}+12 X_{5}+15 X_{6}+12 X_{7}+d_{8}^{-}-d_{8}^{+}=380.000 \\
6 X_{1}+8 X_{2}+7 X_{3}+6 X_{4}+10 X_{5}+8 X_{6}+5 X_{7}+d_{9}^{-}-d_{9}^{+}=200.000 \\
2 X_{1}+2 X_{2}+3 X_{3}+2 X_{4}+1 X_{5}+2 X_{6}+2 X_{7}+d_{10}^{-}-d_{10}^{+}=95000
\end{gathered}
$$

Na função objetivo descrita pela Equação (14), a presença dos pesos $\left(w_{i}^{-}, w_{i}^{+}\right) \geq 0$ faz com que todas as metas de produção de papel fiquem indesejáveis, considerando a possibilidade da solução estar abaixo do valor estabelecido.

$$
\begin{gathered}
\text { MIN }: \frac{w_{1}^{-}}{8000} d_{1}^{-}+\frac{w_{2}^{-}}{2265} d_{2}^{-}+\frac{w_{3}^{-}}{1119} d_{3}^{-}+\frac{w_{4}^{-}}{860} d_{4}^{-}+\frac{w_{5}^{-}}{423} d_{5}^{-}+ \\
\frac{w_{6}^{-}}{19000} d_{6}^{-}+\frac{w_{7}^{-}}{2110} d_{7}^{-}+\frac{w_{8}^{-}}{380.000} d_{8}^{+}+\frac{w_{9}^{+}}{200.000} d_{9}^{+}+\frac{w_{1}^{-}}{95000} d_{10}^{+}
\end{gathered}
$$

Nesse contexto, a Equação (14) apresenta a ponderação relacionada a receita de 380.000 reais, e ponderações relacionadas aos valores acima da meta de custo e consumo de água. A solução da Programação por Metas é viabilizada neste trabalho utilizando Solver/Excel.

\section{Solução do problema e análise de resultados}

A Tabela 1 apresenta as variáveis de decisão do problema juntamente com os desvios percentuais de cada uma. Destaca-se que é possível encontrar diversas formas de solucionar este tipo de problema, porém, todos possuem estruturas similares quanto aos modelos de parametrização. As variáveis de decisão $X_{n}$, onde $n=1, \ldots, 7$, representam os diferentes tipos de papéis a serem produzidos pela empresa e as variáveis $X_{n}$, onde $n=8, \ldots, 10$, estão associadas a receita, custo e consumo de água do processo produtivo. 
Tabela 1 - Variáveis de decisão e desvios

\begin{tabular}{c||c|c|c|c|c|c|c|c|c|c}
\hline \hline Tipo de papel & $X_{1}$ & $X_{2}$ & $X_{3}$ & $X_{4}$ & $X_{5}$ & $X_{6}$ & $X_{7}$ & $X_{8}$ & $X_{9}$ & $X_{10}$ \\
\hline Solução & 0 & 0 & 0 & 0 & 0 & 0 & 0 & 0 & 0 & 0 \\
\hline Desvio under + & 0 & 0 & 0 & 0 & 0 & 0 & 0 & 0 & 0 & 0 \\
\hline Desvio Over - & 0 & 0 & 0 & 0 & 0 & 0 & 0 & 0 & 0 & 0 \\
\hline Metas & 8000 & 2265 & 1119 & 860 & 423 & 19000 & 2110 & 380.000 & 200.000 & 95000 \\
\hline \hline
\end{tabular}

Como pode-se observar na Tabela 2, através do método da Programação por Metas, e seguindo os valores estabelecidos pela direção da empresa, o Solver apresentou uma solução em que a quantidade de cada tipo de papel a serem fabricadas em unidades será de: 8000 papel toalha PIT $\left(X_{1}\right), 2265$ papel toalha PTB $\left(X_{2}\right), 1119$ papel toalha PIM $\left(X_{3}\right), 860$ papel big roll PBR $\left(X_{4}\right), 423$ papel lençol H PLH $\left(X_{5}\right), 13403$ papel higiênico PH $\left(X_{6}\right), 2110$ papel toalha PT $\left(X_{7}\right)$. Seguindo esses valores mostrados no Solver, encontra-se uma receita e custo respectivamente de: $380.000\left(X_{8}\right)$ e 201.114 reais $\left(X_{9}\right)$, com esses valores o lucro será de 178.886 reais.

Seguindo essa indicação de produção, o consumo de água será de 57.055 litros $\left(X_{10}\right)$, estando esse último cerca de $40 \%$ abaixo do consumo total de água estabelecido pela meta de 95.000 litros, logo, a solução aponta para o alcance de grande parte das metas. Contudo, o solver apresentou uma solução em que a quantidade de papel do tipo higiênico PH $\left(X_{6}\right)$ está abaixo da meta estabelecida em $29,46 \%$, e também que a meta relacionada ao custo total $\left(X_{9}\right)$ foi ultrapassada em $0,56 \%$.

Tabela 2 - Primeira solução

\begin{tabular}{c||c|c|c|c|c|c|c|c|c|c}
\hline \hline Tipo de papel & $X_{1}$ & $X_{2}$ & $X_{3}$ & $X_{4}$ & $X_{5}$ & $X_{6}$ & $X_{7}$ & $X_{8}$ & $X_{9}$ & $X_{10}$ \\
\hline Solução & 8000 & 2265 & 1119 & 860 & 423 & 13403 & 2110 & 380.000 & 201.114 & 57055 \\
\hline Desvio under + & 0 & 0 & 0 & 0 & 0 & 5597 & 0 & 0 & 0 & 37945 \\
\hline Desvio Over - & 0 & 0 & 0 & 0 & 0 & 0 & 0 & 0 & 1.114 & 0 \\
\hline Metas & 8000 & 2265 & 1119 & 860 & 423 & 19000 & 2110 & 380.000 & 200.000 & 95000 \\
\hline \hline
\end{tabular}

Vale ressaltar que embora a solução nos mostre um pequeno desvio em relação ao custo da produção em apenas $0,56 \%$, deve-se considerar que um desvio abaixo da meta em $29,46 \%$ na produção de papel higiênico $\left(X_{6}\right)$ pode ser considerado um problema, tendo em vista de que este tipo de produto é um dos que geram uma das maiores receitas dentro da empresa.

Para fazer alterações no cenário inicial de uma solução dada pelo Solver, é importante destacar que cabe ao tomador de decisão a aplicação de seu conhecimento dentro dos critérios das necessidades da empresa. As possibilidades de alteração dos cenários, serão realizados com o objetivo de aprofundar os resultados. Sendo assim, o tomador de decisão considera inviável que a produção de papel tipo higiênico $\mathrm{PH}\left(X_{6}\right)$ esteja $29,46 \%$ abaixo da meta e resolva alterar valores na tabela com o objetivo de satisfazer a meta. Podemos aumentar o valor da ponderação referente a produção de papel higiênico, para o caso de não se alcançar a meta estabelecida.

Conforme apresenta a Tabela 3, ao aumentar a ponderação considerando indesejável não alcançar essa meta de produção de papel higiênico $\left(X_{6}\right)$, o Solver indica que o valor foi alcançado em 19000 unidades de papel do tipo higiênico, atendendo todas as metas de produção em unidades de papel, consequentemente, percebe-se que houveram alterações nos valores de receita e custo. Analisando o novo cenário verifica-se que os valores de receita e custo foram de 463.961 $\left(X_{8}\right)$ e 245.893 reais $\left(X_{9}\right)$ representando, respectivamente, novos desvios acima de $22,10 \%$ e $22,95 \%$, dentro destes parâmetros temos um lucro de 218.068 reais para a empresa. O consumo 
de agua permaneceu abaixo da meta total estando $28,16 \%$ abaixo da meta estabelecida o que pode ser visto como uma excelente perspectiva do ponto de vista ambiental e econômico.

Tabela 3 - Alteração do cenário inicial

\begin{tabular}{c||c|c|c|c|c|c|c|c|c|c}
\hline \hline Tipo de papel & $X_{1}$ & $X_{2}$ & $X_{3}$ & $X_{4}$ & $X_{5}$ & $X_{6}$ & $X_{7}$ & $X_{8}$ & $X_{9}$ & $X_{10}$ \\
\hline Solução & 8000 & 2265 & 1119 & 860 & 423 & 19000 & 2110 & 463.961 & 245.893 & 68250 \\
\hline Desvio under + & 0 & 0 & 0 & 0 & 0 & 0 & 0 & 0 & 0 & 26750 \\
\hline Desvio Over - & 0 & 0 & 0 & 0 & 0 & 0 & 0 & 83.961 & 45.893 & 0 \\
\hline Metas & 8000 & 2265 & 1119 & 860 & 423 & 19000 & 2110 & 380.000 & 200.000 & 95000 \\
\hline \hline
\end{tabular}

Também destaca-se que a solução embora tenha se desviado acima tanto em receita como em custo, a mesma aponta para um aumento de lucro para essas novas condições de produção de: 39.182 reais a mais em relação a primeira solução, subtraindo a primeira receita gerada de 218.068 reais da segunda cujo o valor encontrado foi de 178.886 reais.

Por isso, é importante destacar que na PM é necessário que se faça na maioria dos casos mais de uma simulação e análise dos novos resultados. Embora as metas de custo e receita tenham sido alteradas mediante as ponderações atribuídas, novas soluções possibilitaram maior lucratividade, logo, no exemplo estudado verifica-se que trabalhar com outros cenários forneceram ao tomador de decisão mais detalhamento da solução 1 em detrimento da solução 2.

Porém, é importante ressaltar novamente que tudo depende do posto de vista e análise do gestor, para cada caso há uma necessidade diferente. Se aplicarmos conceitos ou técnicas de gestão e modelos de estastísca, as possibilidades utilizando a técnica de Programação por Metas são infinitas, até que o tomador de decisão considere a solução satisfatória para a aplicação.

\section{Conclusão}

A preocupação com a tomada de decisão nos setores industriais conduz de forma geral a sistemas multiobjetivos, pois o processo decisório nesses casos agrega diversos níveis de decisão, quase sempre intercalados. A Programação por Metas vem se mostrando nas suas mais diversas abordagens, como uma metodologia interessante para subsidiar no equilíbrio dos objetivos a serem otimizados, possibilitando certa margem de manipulação (através das ponderações) ao gestor industrial.

Neste trabalho foram apresentados a modelagem matemática, solução por meio do software Solver/Excel e uma análise sobre uma aplicação da Programação por Metas Ponderadas em um problema de decisão associado ao processo produtivo de papéis sanitários, elencando um cenário em que estavam envolvidas metas de produção, receita e sustentabilidade. Pertubando a função objetivo do modelo, através dos parâmetros de ponderação, e observando o conjunto de taxas de variação das metas, foi possível obter um nível satisfatório de otimização.

\section{Referências}

BITTENCOURT, E. Parâmetros de otimização no processo de fabricação de celulose e papel. 2004. 61 f. Dissertação (Mestrado em Engenharia Florestal) - Pós-graduação em Engenharia Florestal, Setor de Ciências Agrárias, Universidade Federal do Paraná, Curitiba, 2004. 
CAMPELLO, B. S. C. et al. Otimização do problema de dimensionamento de lotes integrado ao problema de corte de estoque bidimensional em uma indústria de papel. In: ENCONTRO REGIONAL DE MATEMÁTICA APLICADA E COMPUTACIONAL, 4., 2017, Bauru. Caderno de trabalhos completos e resumos. Bauru: Unesp, Faculdade de Ciências, 2017. p. 512-514. Disponível em:<http://www.fc.unesp.br/\#!/departamentos/matematica/eventos 2341 /ermac/cadesnos-de-trabalhos-completos-e-resumos/>. Acesso em: 12 out. 2017.

DAN, E. D.; DESMOND, O. Goal programming: an application to Budgetary allocation of an institution of higher learning. Research Journal in Engineering and Applied Sciences, v. 2, n. 2, p. 95-105, 2013.

INDÚSTRIA BRASILEIRA DE ÁRVORES. Iba: 2015. Disponível em: $<$ http://iba.org/images/shared/iba_2015.pdf $>$. Acesso em: 20 jul. 2016.

NALI, E. D.; RIBEIRO, L. B. N. B.; DA HORA, A. B. Biorrefinaria integrada à indústria de celulose no Brasil: oportunidade ou necessidade? BNDES Setorial. v. 43, p. 257-294, 2016. Disponível em: $<$ https://web.bndes.gov.br/bib/jspui/bitstream/1408/9578/1/BS/\%2043 $\% 20$ Biorrefinaria\%20integrada\%20\%C3\%A0\%20ind\%C3\%BAstria\%20de\%20celulose \%20no\%20Brasil\%20_PB_D.pdf>. Acesso em: 10 jul. 2016.

RAGSDALE, C. T. Managerial decision modeling. 9. ed. Mason: Cengage Leaning, Inc, 2011. p. 306.

SCHNIEDERJANS, M. J. Goal programming: methodology and applications. Springer Science, 1995.

SEN, N.; NANDI, M. Goal programming, its application in management - special attention into plantation management: a review. International Journal of Scientific and Research Publication, v. 2, n. 9, p. 1-6, 2012.

SILVA, C. A. F.; BUENO, J. M.; NEVES, M. R. A indústria de celulose e papel no Brasil na primeira década do século XXI: algumas considerações sobre o que poderá ainda acontecer.

Guia ABTC Fornecedores \& Fabricantes: celulose e papel, 2015. Disponível em: $<$ http://www.eucalyptus.com.br/artigos/2015_ABCT_Panorama_Setorial.pdf $>$. Acesso em: 25 jun. 2016.

VASCONCELOS, D. V.; POLTRONIERE, S. C. Estudos sobre o problema de corte de estoque unidimensional com aplicação na indústria de papel. In: ENCONTRO REGIONAL DE MATEMÁTICA APLICADA E COMPUTACIONAL, 4., 2017 Bauru. Caderno de trabalhos completos e resumos. Bauru: Unesp, Faculdade de Ciências, 2017. p. 474-476. Disponível em: $<$ http://www.fc.unesp.br/\#!/departamentos/matematica/eventos2341/ermac/cadesnos-detrabalhos-completos-e-resumos/>.

Acesso em: 13 out. 2017.

Artigo recebido em jun. 2017 e aceito em dez. 2017. 